Territorios 37 / Bogotá, 2017, pp. 41-59

ISSN: 0123-8418

ISSNe: $2215-7484$

Ciudades y conflictos en América Latina: ayer, hoy, mañana (II)

\title{
Tegucigalpa a través de los conflictos por la primacía urbana en Honduras, s. XVIII-XX *
}

Tegucigalpa through the Conflicts for the Urban Primacy in Honduras, s. XVIII-XX

Tegucigalpa através dos conflitos pela primazia urbana na Honduras, s. XVIII-XX

\section{Daniela Navarrete Cálix ${ }^{* \star}$}

Recibido: 1 de mayo de 2016

Aprobado: 27 de abril de 2017

Doi: http://dx.doi.org/10.12804/revistas.urosario.edu.co/territorios/a.4846

Para citar este artículo:

Navarrete, D. (2017). Tegucigalpa a través de los conflictos por la primacía urbana en Honduras (s. XVIII a XX). Territorios, (37), 41-59. Doi: http://dx.doi.org/10.12804/revistas.urosario.edu.co/territorios/a.4846

* El presente artículo es parte de la tesis de doctorado (en curso): Tegucigalpa, laboratorio urbano de las modernidades en Hondurass. XIX y XX realizada bajo la dirección de Alain Musset en la Escuela de Estudios Superiores en Ciencias Sociales (EHESS), París.

** Master en Investigación en Ciencias Sociales de la Escuela de Estudios Superiores en Ciencias Sociales (EHESS), Paris. Profesora asociada de Historia. Es cuela Agrícola Panamericana Zamorano, Honduras. Correo electrónico: dnavarrete@zamorano.edu ORCID: http://orcid. org/0000-0002-6090-3688 
Palabras clave

Conflicto, jerarquía urbana, modernidad, primacia, identidad local.

Keywords

Conflict, urban hierarchy, modernity, primacy, local identity.

Palavras-chave

Conflito, hierarquia urbana, modernidade, primazia, identidade

local.

tersitarias 37

42
RESUMEN

El presente es un estudio con enfoque histórico-cronológico de larga duración que busca responder a la cuestión sobre el impacto que generaron y generan los conflictos por la primacía urbana en Honduras. Este concepto, propio de la geografía urbana, se articula transversalmente con los eventos que manifestaron esa conflictividad, a saber: cambios en la jerarquía urbana, en las relaciones socio-históricas, ciclos de economía y la evolución de los transportes. Para ello abordaremos tres momentos claves de la conflictividad: la lucha por la restitución de la Alcaldía Mayor de Tegucigalpa durante el Régimen de Intendencias (1788-1812), la pugna por convertirse en la capital del país en el proceso de invención del Estado-Nación (1821-1880) y la dicotomía urbana ante San Pedro Sula (1920-1990). Esta última, epicentro de los proyectos económicos liberales y neoliberales del país.

\section{ABSTRACT}

The present is a long-term historical-chronological study that seeks to answer the question about the impact generated in the past and present times by the conflicts over urban primacy in Honduras. This concept, typical of urban geography, articulates transversally with the events that manifested this conflict, namely: changes in the urban hierarchy, socio-historical relations, cycles of economy, transport evolution. To this end, we will address three key moments of conflict: the fight for the restitution of the Alcaldía Mayor of Tegucigalpa in the regime of Intendencias (1788-1812), the struggle to become the capital of the country in the process of invention of the Nation-State (1821-1880) and the urban dichotomy against San Pedro Sula (1920-1990). The latest, epicenter of liberal economic and neoliberal economic projects in the country.

\section{RESUMO}

O presente é um estudo com enfoque histórico-cronológico de longa duração que busca responder à questão sobre o impacto que geraram e geram os conflitos pela primazia urbana na Honduras. Este conceito, próprio da geografia urbana, articula-se transversalmente com os eventos que manifestaram essa conflitualidade, a saber: mudanças na hierarquia urbana, nas relações sóciohistóricas, ciclos de economia e a evolução dos transportes. Para isto, abordaremos três momentos chave da conflitualidade: a luta pela restituição da Prefeitura Maior de Tegucigalpa no regime de Intendências (1788-1812), a pugna por converter-se na capital do país no processo de invenção do Estado-Nação (1821-1880) e a dicotomia urbana ante San Pedro Sula (1920-1990). Esta última, epicentro dos projetos econômicos liberais e neoliberais do país. 


\section{Introducción}

Esta pesquisa proviene de un estudio comparativo más amplio sobre el impacto de la modernidad en la capital de Honduras en el período liberal y neoliberal. El enfoque ahí es el de una comparación diacrónica que, sin embargo, requirió una construcción histórico-cronológica de larga duración de Tegucigalpa, a fin de captarla en su dimensión evolutiva. A medida que se profundizaba en el evento del cambio de capital, surgió la relevancia que generaron y generan los conflictos por la primacía urbana, fenómeno que se ha dado en varios lugares del mundo: Brasil, India y, en el caso de Centro América, en tres países: Costa Rica, Nicaragua y Honduras. Este concepto, propio de la geografía urbana, se articula transversalmente con los eventos que manifestaron esa conflictividad, a saber: cambios en la jerarquía urbana, en las relaciones socio-históricas, ciclos de economía y la evolución de los transportes. El objetivo aquí es el de reconstruir las relaciones que opusieron a los poderes locales y provinciales en la medida que inciden en los anhelos políticos de sus élites, en contextos de intereses más amplios de Estado-Nación o proyectos económicos globales. Sin duda una propuesta muy ambiciosa para este espacio conciso, por lo que nos centraremos en resaltar tres momentos clave del proceso urbano hondureño: siglos XVIII, XIX y XX. ¿Qué cambió en el fondo de las disputas por la primacía urbana en los tres momentos? La escala planteada es la nacional en razón de que el modelo urbano moderno y sus conflictos se iniciaron con el establecimiento de la provincia de Honduras en el s. XVI y continuaron después en el Estado hondureño. Ello no impide, por supuesto, las comparaciones con otros casos regionales cuando es pertinente.

En cuanto al tratamiento del tema, hemos procurado sustentarnos en los análisis histórico-urbanos que se han hecho para el orbe indiano, como diría Brading (1991). Este recorrido nos ha llevado a la comprensión de la visión de textos fundadores como los de urbanismo en Hispanoamérica de Francisco de Solano (1987), la evolución del pensamiento mestizo de Gruzinsky (1999) o las consideraciones por la globalización urbana de Davis (2005), por mencionar algunos. Una visión más precisa sobre la región nos la procuran las investigaciones sobre jerarquía urbana en Centro América (Musset, 2011), las emociones alrededor de la mudanza de una capital latinoamericana (Vidal, 2009) y las disputas entre ciudades rivales en países vecinos (Kinloch, 2005). Para Honduras, los estudios son escasos por lo que partimos de las fuentes bibliográficas más consistentes como investigaciones económicas de la sociedad colonial (MacLeod, 1976), las geografías regionales sobre las ciudades protagonistas de esta investigación, Comayagua y Tegucigalpa (Reina Valenzuela, 1980; Martínez, 1982; Barahona, 1993). Estos análisis son complementados con el de fuentes primarias en los archivos 
hondureños, municipales y nacionales, lo que nos permitió enriquecer y consolidar interrogantes en base a los hallazgos hechos. Las particularidades del caso hondureño son, a saber: la preminencia del peso económico sobre el político en los tres momentos estudiados, así como la desaparición del modelo primacía urbana en el último momento analizado.

Estudiar el fenómeno de las primacías urbanas implica analizar y entender las relaciones entre ciudad, territorio y redes urbanas. El espacio colonial del actual territorio hondureño se constituyó entre tres ejes: noroeste-occidente, centro-sur y nororiente-oriente. La red primaria de centros urbanos se completó antes de terminar el siglo XVI y perduró todo el período colonial. Solano clasificó los centros urbanos fundados según su tamaño y función, elementos que contribuyen con su rango o estatuto. Por su función había centros político-administrativos (como las sedes virreinales, de audiencias y gobernaciones), centros militares, mineros (como los reales de minas) y puertos. Por su tamaño e importancia, las leyes establecían la jerarquía en ciudades, villas, reales de mina y pueblos de indios. En Honduras, las primeras fundaciones fueron puertos $\mathrm{y}$, a medida que se colonizaba el interior, aparecieron reales de minas, centros administrativos, militares y ganaderos que podían ser villas o ciudades (Solano, 1987). La vida urbana en Indias no es privativa de los españoles, los pueblos de indios también fueron objeto en alguna medida de estas reconfigu- raciones. El sentido que tenía en esa época el estatuto de las ciudades, o el "deseo de ciudad" de los españoles como señalan las investigaciones de Calvo (1995) y Musset (2011), se debe medir tomando en cuenta la posición de privilegio, extendida tanto para los centros urbanos como para los habitantes. Lo anterior es evidente en las Nuevas ordenanzas de descubrimiento y población de 1573 , donde la Corona se comprometía a ennoblecer a los vecinos de ciudades americanas con el rango nobiliario de "hijosdalgo de solar", que significaba eran sujetos de gozar "todas las honras y preheminencias [sic]" (Musset, 2011). El orgullo familiar de descender de unos hijosdalgo, ligado a la posesión de solar, son el origen del fuerte arraigo al terruño y posterior identidad local.

En el contexto de las relaciones interurbanas y su articulación en regiones, desde muy temprano se manifestaron en Honduras conflictos por la primacía urbana. En el siglo XVI esa disputa recayó sobre el puerto de Trujillo (f. 1524), primera sede de la gobernación de Honduras, San Pedro de Puerto Caballos, sede de la Caxa Real, Gracias y Comayagua, sedes consecutivas de la administración política. Esa disputa se resolvió a favor de Comayagua a mediados el siglo XVI (villa en 1533 y ciudad en 1557), que concentró las sedes políticas y eclesiásticas más importantes en la provincia: la gobernación y el episcopado. Para una jurisdicción alejada como la de Honduras, la sede de la gobernación era el rango más alto al que podía aspirar

\section{tersitarias 37}


una población. La primacía urbana repercutía directamente en la construcción simbólica de la ciudad, pues la urbe hegemónica concentraba las obras públicas más importantes para el gobierno y habitantes. Así, Comayagua concentró elementos representativos de la modernidad urbana renacentista, con su plano en ajedrez, su arquitectura religiosa (catedral, iglesias, colegio eclesiástico, hospital y ermitas), civil (gobernación, cabildo, caxa real, cuartel de dragones) y casas de notables a la usanza de la península. También articuló los pueblos de indios, dejando manifiesto la presencia de un orden, que es a la vez integrador y desigual.

Transcurridas varias décadas desde que Comayagua es capital de provincia y ciudad, se funda a $80 \mathrm{~km}$ al sur el real de minas de Tegucigalpa (1578). La montañosa Tegucigalpa inicia su construcción histórica-urbana como un campamento minero español o real de minas, hasta devenir villa (1767), ciudad (1821) y capital de la República (1880). El motor de la economía es la minería, moderada pero suficiente para permitir subsistir a mineros, comerciantes, hacendados blancos y un mosaico étnico de mano de obra mestiza, india y negra. Al lado de las otras ciudades y villas de la provincia, Tegucigalpa detenta una posición periférica y secundaria respecto su red urbana inmediata. Sin embargo, la creación de la Alcaldía mayor de minas de Honduras en 1580, favoreció su consolidación como el centro minero de más importancia en Honduras y probable- mente el más rico de la Capitanía General de Guatemala, configurando un cuerpo autárquico bien articulado desde el punto de vista geográfico, económico y político. Así lo favoreció la Capitanía en Guatemala al cederle de su propia jurisdicción, la villa de Choluteca, un próspero centro agrícola-ganadero y minero, sus pueblos de indios y territorios que se extendían hasta la Bahía de Fonseca, en el Pacífico. Desde su creación, la Alcaldía Mayor de Tegucigalpa fue dotada para su autogobierno, con autoridades propias, Alcalde Mayor, tribunales de justicia, oficiales de la Hacienda Real y un juez de minas. Esta condición de autarquía estuvo al origen del primer conflicto urbano que le enfrentó a Comayagua y que abordaremos más adelante.

La ubicación de Tegucigalpa al sur de la provincia, favoreció su inserción en la red comercial del istmo que discurría a lo largo de la planicie costera del Pacífico, a través de ferias regionales en el sector norte, que unía a San Salvador, Chiapas, Oaxaca y Veracruz. Sucedió lo mismo con el sector sur (Nicaragua, Costa Rica y Panamá). Esta vinculación económica en tres niveles (el local minero, el regional agrícola-ganadero y el suprarregional comercial) va a desplazar el eje económico del norte-centro de la provincia (articulado desde los 1530 por Puerto Caballos y Comayagua) al centro-sur, articulado por $\mathrm{Te}$ gucigalpa y Choluteca (MacLeod, 1976). Esta incipiente red urbana (ver figura 1) se consolidó paulatinamente. 


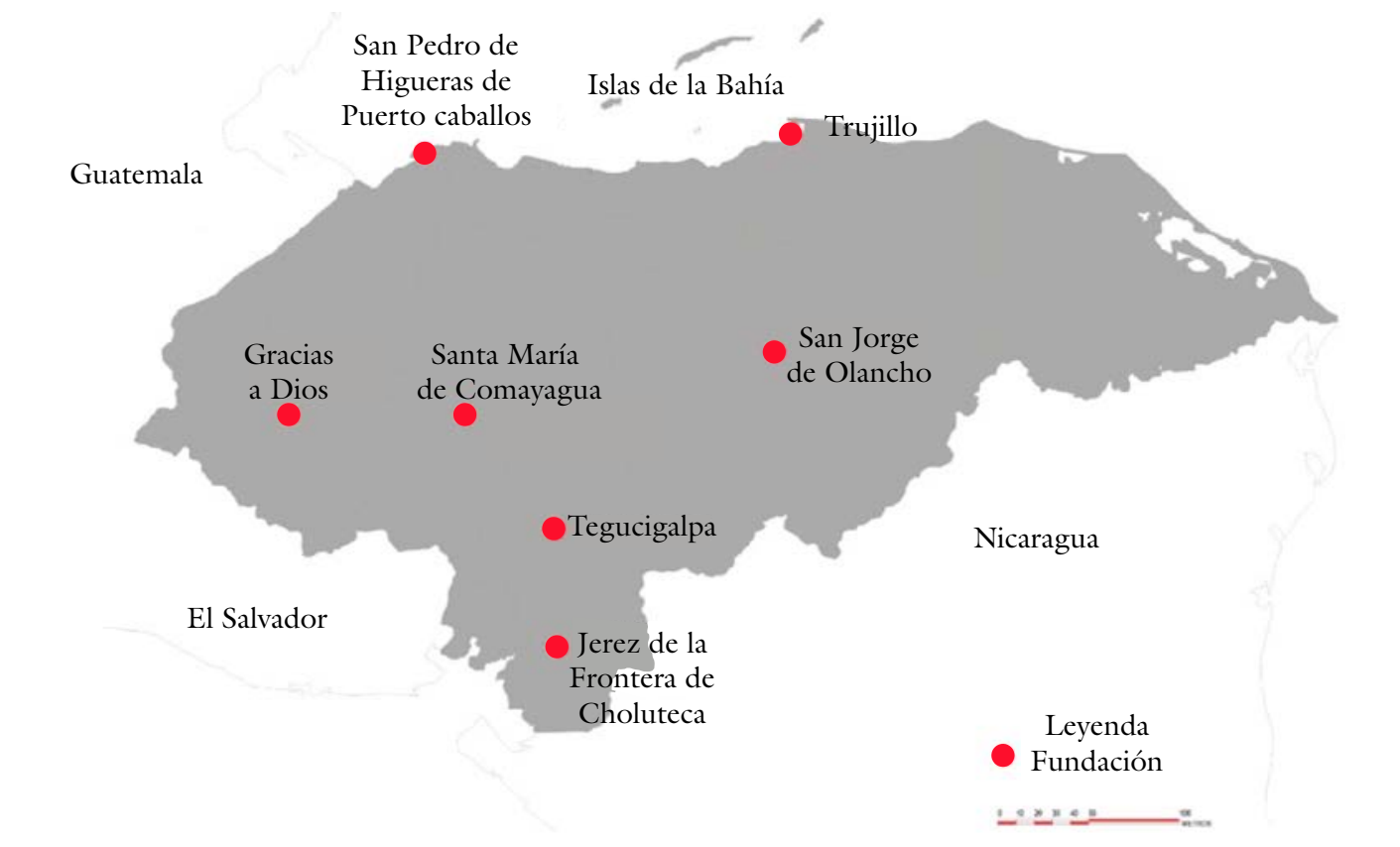

Fuente: elaboración propia, 2016

\section{La lucha por la restitución de la Alcaldía Mayor de Tegucigalpa frente a la Intendencia de Comayagua, capital provincial (1788-1812)}

El antecedente del primer conflicto se sitúa a inicios del siglo XVIII, cuando el Real de Minas de Tegucigalpa experimentó un nuevo ciclo de dinamismo económico. La bonanza minera y comercial, junto con la práctica de la ganadería, le permitió a su vecindario alcanzar, para finales del siglo territarias 37 XVIII, cerca de siete mil habitantes (Vallejo, 1997). Esto condujo a la visita del
Capitán General de Guatemala, Alonso Fernández de Heredia, quien elevó Tegucigalpa a la categoría de villa, el 10 de junio 1762. A partir de la adjudicación del título de villa, Tegucigalpa fue dotada de cabildo, institución política local que jugó un rol fundamental en la construcción de las aspiraciones políticas de las élites minero-comerciales asentadas en el poblado. Seis años después, en Real Cédula del 17 de julio de 1768 fue confirmado el nombramiento como Villa:

[...] por lo distinguida que es aquella población de ricos y abundantes Minerales [...] 
los relebantes [sic] motivos de ser Capital de la Provincia, abundante de ganados y géneros con que sus naturales acuden anualmente a la feria de cerro redondo, muchos minerales que cuasi sobstienen [sic] la labor de la Real Casa de la Moneda de la Ciudad de Guatemala, las innumerables cantidades de quintos y consumo de azogues que han rendido a mi Real Hazienda (Real Cédula, 1768 en Taracena, 1998, p. 205 $)^{1}$.

En la cita anterior se enuncia lo que fue la manzana de la discordia entre Comayagua y Tegucigalpa. Al destacarse en la confirmación de la nueva villa, la existencia de relebantes [sic] motivos para ser capital de la provincia, la pérdida de la primacía urbana fue a partir de ahí una amenaza real y concreta para Comayagua y así lo entendieron sus autoridades. Por su parte, las familias tegucigalpenses consolidan sus redes en el poder económico, político y eclesial, pues son dueñas de los medios de producción (haciendas y minas) y de cargos político-religioso (Martínez, 1989). El cabildo no solo estructura la organización administrativa, sino que además es un trampolín político para la élite que perdurará a través de redes familiares en el período republicano. El nuevo estatuto trajo nuevas unidades urbanas, como la Caxa Real y el Cabildo; más templos y ermitas con sus plazas en estilo barroco. El criterio de jerarquía socio-racial se reflejó en las casas de las élites levantadas en el núcleo de la comuna y alrededor de sus plazas. En tanto, los pueblos de indios de Tegucigalpa se encontraban en los márgenes o al otro lado del río Choluteca.

Las nuevas castas proliferaron desde el siglo XVIII. El más emblemático asentamiento fue el barrio de pardos y mulatos de Los Dolores, al oeste de la villa. Sus pobladores llegaron a ser tan prósperos como los mineros españoles al punto que construyeron la iglesia más grande de la villa.

En la segunda mitad del siglo XVIII se implementan las Reformas Borbónicas, mecanismo de centralización y racionalización burocrática de la administración española. Se crean las Intendencias, cuyo modelo fue el cargo de los intendentes franceses. Estas tenían facultades judiciales y en parte también militares, su objetivo era mejorar la fiscalidad. Se disuelven así las viejas provincias y sus sub-divisiones: los corregimientos y las Alcaldías mayores, dividiéndose la Intendencia en partidos, a cargo de un sub-delegado. En la provincia de Honduras se aplican en 1788, suprimiéndose la Alcaldía Mayor de Tegucigalpa y subordinándola como partido a la Intendencia de Comayagua. Se ha señalado que la imposición del régimen de intendencias, desconociendo la tradición política, económica y social de Tegucigalpa, significó la interrupción de un ciclo productivo (Barahona, 1993). Por ejemplo, los trabajadores del mineral de Yuscarán, antes bajo la Alcaldía Mayor, se quejan de la falta de sal, indios y plomo para la extracción minera, pues la atomización de la Alcaldía en partidos de Comayagua centralizó las operaciones haciendo la medida antieco- 
${ }^{2}$ Las cursivas son propias.

\section{territarias 37}

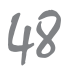

nómica e inviable administrativamente. La pérdida de su rango regional significó un golpe político de mayores repercusiones a corto, mediano y largo plazo para la Villa. En corto plazo, se subordina la jurisdicción territorial de la Alcaldía Mayor, incluida la villa de Tegucigalpa a la Intendencia de Comayagua. A mediano plazo, esta decisión trunca las aspiraciones en convertirse en ciudad y, peor aún, el equilibrio político local es destrozado. A largo plazo, va a desencadenar una aversión a Comayagua (y por extensión a la Corona) lo que llevará a formular reivindicaciones independentistas. Ante la nueva situación, los grupos económicos tegucigalpenses más importantes se aglutinaran para recuperar por todos los medios la libertad de maniobra administrativa de la que habían gozado desde hacía dos siglos. Esta libertad en la práctica significó por parte de los mineros tegucigalpenses, el incumplimiento de las prohibiciones oficiales sobre explotación indígena y el fraude en el uso del azogue. La oposición al sistema de Intendencias, por tanto, fue una reacción lógica pues el nuevo orden administrativo buscó terminar con prácticas arraigadas en perjuicio de la Corona. La respuesta de la villa fue nombrar al Procurador General Francisco Albert en 1799 para que, en nombre de los vecinos, peticionase ante la Corona la desagregación del partido de la Intendencia de Comayagua (Martínez, 1982).

Los intentos de Tegucigalpa por desagregarse de Comayagua y restituir la $\mathrm{Al}$ caldía Mayor duraron trece años. Pronto unieron otra petición: la de otorgar a la villa el título de ciudad. En el acta capitular del 24 de septiembre de 1806 se señala el envío de siete cartas al rey exponiendo los problemas de distancia con Comayagua. Se menciona también el ofrecimiento de donar "a su magestad [sic] dos mil pesos en plata fuerte puestos en las reales caxas [sic] de Guatemala" (Acta capitular, 1806) para dicho efecto de "desagregar la provincia y concederle el título de ciudad como las demás de Indias" (Acta capitular, 1806). ¿Era tan importante del estatuto urbano para la población americana en el ocaso de la administración española? El ofrecimiento abierto al rey por la desagregación y elevación a ciudad a cambio de un precio, revela que el paso del tiempo no ha mermado el deseo de ciudad, "como las demás de Indias". En otra petición enviada a la corona en 1807 el Procurador de la villa expone que:

[...] no es menos constante que se la degradó [a la Alcaldía Mayor de Tegucigalpa] desde la creación de Yntendencias [sic] en el año de 1788 por haberse avocado [ sic] asi la de Comayagua toda su jurisdicción $[. .$.$] con el$ gravamen de surtir de carnes a la nueva capital [de provincia, Comayagua] y con otros arbitrios honerosos [sic] para aumentar sus propios incompatibles ciertamente con las ventajas de aquella villa y por de contado en perjuicio también de los intereses de $\mathrm{V}^{\mathrm{a}}$. $\mathrm{R}^{\mathrm{a}}$ hacienda ${ }^{2}$.

Grosso modo, la percepción de los tegucigalpenses sobre el régimen de Intendencia se simplificaba a pérdida de soberanía 
sobre su jurisdicción y sobre sus bienes. En una palabra: el corazón de su identidad histórica local.

En ese contexto se suscitó la ocupación francesa del trono español entre 1808 y 1812. Una vez restaurado Fernando VII, la Corona, deseosa de encontrar aliados en tiempos en que proliferaban las conspiraciones separatistas, restauró la Alcaldía Mayor. El hecho consolidó en los tegucigalpenses el alto concepto que tenían de sí mismos y del peso que Tegucigalpa tiene de hecho, según ellos, en la región y la provincia. El restablecimiento de la Alcaldía Mayor desató las reacciones en contra de la Intendencia de Comayagua. Desde 1815 datan los informes de los gobernadores intendentes que expresan la ruina en que se encuentra la villa en todos los sentidos, notoriamente el financiero. Para el gobernador Antonio de Tornos, esto se debe a la desobediencia de la Alcaldía a las providencias emitidas por la Intendencia de Comayagua y a los "continuos pleitos y parcialidades en que se haya sumergida dicha Villa desde la segregación de la Intendencia" (de Tornos, 1815), haciendo quedar a los tegucigalpenses como incapaces de gobernarse. En 1820, otro Gobernador Intendente, José Gregorio Tinoco de Contreras, en otro informe enviado al rey de España, señala como causa del atraso de la provincia la separación del partido de Tegucigalpa y solicita la supresión de la Alcaldía Mayor "pues no es más que un traspantojo de los mineros de Tegucigalpa, que más debían dedicarse a la agricultura que a la saca de minerales que solo les produce para pasar el día” (Tinoco, 1820) quedando claro que la solución el problema de la desagregación es la supresión de la Alcaldía Mayor. Las descalificaciones verbales fueron acompañadas por persecuciones políticas. Una vez restablecida la Alcaldía Mayor de Tegucigalpa, las autoridades de la Intendencia de Comayagua acusaron de malversación de fondos a Manuel José Midence Zelaya. Este era un criollo perteneciente a uno de los grupos familiares más importantes de la villa, era regidor perpetuo, teniente de ministros y uno de los principales artífices de la restauración de la Alcaldía. Desde 1813 se había comenzado a investigar su gestión como encargado de la Caja de Rescates (1806 al808). En el proceso, que duró de 1814 a 1820, se le separó del cargo de sub-intendente. Adicionalmente, toda la familia Midence fue marginada de cargos administrativos y políticos. La acusación no se comprobó pues no hubo penas ni multas (Zepeda, 2006). El mismo autor sostiene que el juicio tenía por objetivo político neutralizar la figura de Manuel José Midence. Paradójicamente el caso fortaleció el espíritu antimonárquico de los criollos de Tegucigalpa. Tras la muerte del Alcalde Mayor Narciso Mallol en 1821, le remplazará en el cargo con carácter interino José Tomás Midence, hermano de Manuel José. El 15 de septiembre del mismo año se realiza la independencia, cuyos pliegos llegaron el 28 del mismo mes a la villa. El cabildo firmó la adhesión a la Independencia "con gran solemnidad en el marco de una gran fiesta popular” (Zepeda, 2006, p. 101). territarias 37

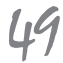




\section{Continuidad del conflicto por la capital del país (1821-1880)}

En represalia a la rebeldía tegucigalpense, las autoridades españolas en Comayagua no promovieron el ascenso de estatuto a ciudad. Pero esto ya no tenía importancia dado el fin del pacto colonial, desde entonces los pueblos y sus cabildos asumen su soberanía y se inicia el proceso de centralización de las naciones modernas. El título de ciudad llegó fulminante a los tres meses de la independencia, el 11 de diciembre de 1821, la Junta Consultiva de Guatemala emite el acuerdo que dice:

[...] teniendo presente el patriotismo que ha acreditado el muy noble Ayuntamiento de la Villa de Tegucigalpa, desde la época de nuestra gloriosa independencia $[\ldots]$ y el rango que ocupa en la escala de las poblaciónes de aquella provincia, se acordó: Que en lo sucesivo se le dé a la Villa el título de ciudad, y a su ayuntamiento el de patriótico (Junta Consultiva de Guatemala, 1821 en Vallejo, 1997, p. 17).

En el anterior se ratifica el doble carácter del título de ciudad: el que recae sobre la ciudad (urbs) y sobre su gobierno (civitas). El centro de gravedad del poder se desplaza de Madrid a Guatemala, bajo el control de liberales y quienes retribuyeron a las élites tegucigalpenses. El nuevo estatuto de ciudad fortalece el liderazgo de la misma en la región, por encima de comunas que ya tenían ese rango, como Choluteca, al sur y Danlí, al oriente. Tras la anexión a México, en 1824 se creó el nuevo país, las Provincias Unidas de Centro América, también conocido como la Federación. Esta etapa supuso un nuevo ciclo de rivalidades urbanas, en un contexto regional de pugnas entre visiones de proyectos liberales radicales y moderados. El conflicto se manifestó al momento de definir la sede del Congreso constituyente para promulgar la constitución del Estado de Honduras. Comayagua continuó siendo capital ahora nacional, con el desacuerdo de Tegucigalpa. Para atenuar las cosas, se decidió por una tercera localidad menor y sin pretensiones, el mineral de Cedros, al norte de Tegucigalpa. Se declaró en esa misma ocasión que la capital se alternaría entre las dos, modalidad que no llegó a cumplirse. Este conflicto urbano es el reflejo de la contradicción del ideal de modernidad política anhelado (el Estado Nación) y la realidad de múltiples comunidades heterogéneas dotadas de arraigo (la llamada patria chica). Tal contradicción, señala Guerra, presentó a los sectores gobernantes del periodo post-independentista un doble desafío: crear, en primer lugar, un Estado centralizado y, luego, a partir de éste, inculcar en los ciudadanos individuales un sentido de lealtad hacia el nuevo centro de poder (Guerra, 1993). La creación de un Estado centralizado implicaba la subordinación de las lealtades tradicionales, proceso que enfrentó una fuerte resistencia durante todo el siglo XIX, por parte de los órganos de poder local. Esta polarización incidió en territorios y ciudades por toda América Latina. Quito, conservadora y 
Guayaquil, liberal, fueron buen ejemplo de ello en Sur América. En Centro América, por provincia se enfrentaron en Nicaragua por veinte años, León y Granada; en Costa Rica, Alajuela y San José contra Heredia y San Salvador contra Guatemala. En el caso nicaragüense, como lo señala Kinloch, las ciudades de León y Granada adoptaron distintas posiciones luego de la Independencia. La primera decidió proclamar su anexión al Imperio Mejicano, mientras la segunda, conservando su lealtad a Guatemala, formó su propia Junta Gubernativa republicana. Esta oposición profundizó antiguas pugnas localistas, desembocando en una cruenta guerra civil que se prolongó de 1823 a 1825 primero y una nueva y más cruenta guerra civil a fines de 1844 . El conflicto reflejó la gravedad de las tensiones generadas en el proceso de incorporación de las comunidades políticas del Antiguo Régimen, dentro del modelo ideal de nación propio de la modernidad (Kinloch, 2005).

En la década de 1850, surgen las propuestas de grandes obras para impulsar el progreso económico del país. Es la época de industrialización europea y norteamericana y los proyectos de comunicación son cruciales. El encargado de negocios norteamericano E. G. Squier dedicó especial atención al Valle de Comayagua en razón del interés de llevar a cabo la construcción de un ferrocarril interoceánico entre Puerto Caballos y la Bahía de Fonseca. Este paso es vital para acortar la comunicación entre la costa este y oeste estadounidense, especialmente tras los descubrimientos de oro en California. El diplomático conoce las observaciones y motivaciones de los españoles al fundar la ciudad de Comayagua contenidas en la narración de Juarrós, el historiador del Reyno de Guatemala:

[...] a mitad de camino entre los dos océanos [...] con el propósito de obtener una fácil comunicación entre el Atlántico y el Pacífico con la cual se evitarán tantas enfermedades y pérdidas de vidas humanas y muchas fatigas y privaciones que se padecían en la travesía de Nombre de Dios (Chagres) a Panamá (Squier, 1971, p. VI).

El fiasco del proyecto del ferrocarril ${ }^{3}$ trajo la frustración del proyecto de progreso del país y de Comayagua, pues las malas condiciones de las comunicaciones terrestres contribuyeron, entre los factores ya mencionados, a su decline inminente. Para 1854, la población de Comayagua apenas llega a dos mil. Durante la administración de Céleo Arias (1872 a1874) la ciudad fue nuevamente sitiada. Este conflicto destruyó parte de la ciudad y provocó un éxodo masivo:

[... la guerra que Guatemala y El Salvador trajeran a Honduras en 1873 y que concluyó por sitiar la ciudad, saquear multitud de casas,después de arruinadas motivó también nueva emigración, que se aumentó en grande escala cuando en 1880 se decretó que la ciudad de Tegucigalpa sería la residencia del Gobierno (Vallejo, 1997, p. 26).

De igual forma, la economía de la ciudad se redujo casi en exclusiva a las acti-
${ }^{3}$ El proyecto del ferrocarril interoceánico fue iniciado a través de préstamos que el gobierno de Honduras contrajo con la banca inglesay francesa. El proyecto apenas se extendió unos kilómetros desde Puerto Cortéshacia el interior, sin ser completado nunca debido, principalmente, al mal manejo de los fondos. 
vidades derivadas de las funciones políticoadministrativas. La suerte estaba echada. La pérdida de la capitalidad de Comayagua tuvo dos resultados: primero fue el inicio del fin de la rivalidad entre ambas ciudades que databa desde tiempos coloniales y segundo desencadenó su estancamiento que se prolongaría varias décadas. $\mathrm{Al}$ respecto, la inglesa Mary Lester describe la situación en 1881:

[Comayagua] está construida pintorescamente, pero sus calles silenciosas cubiertas de polvo, su aire de pobreza y la ausencia de una vida agitada y activa, todo anuncia que su gloria es cosa del pasado. Hay [sin embargo] muchos celos de Tegucigalpa, donde el presidente vive ahora (Lester, 1971, p. 146).

Según el antropólogo Steven Reif (1980), la rivalidad entre las ciudades continuó pero de manera más reducida. Comayagua ya no podía competir y, en consecuencia, el complejo localista tomó mayor importancia. Este servía como mecanismo de defensa contra el impacto del poder político y económico de la nueva capital. Las dificultades de comunicaciones propiciaron el aislamiento de los pobladores y facilitaron mantener un aura de prominencia nacional. Aunque este localismo ha disminuido con los años, es característico de los comayagüenses. Ellos identifican hoy el centro de la ciudad como su patrimonio histórico, como el vestigio del esplendor de un pasado glorioso y lejano.

\section{tersitarias 37}

hasta hoy precisamente por la extinción del empuje modernizante en la antigua capital de provincia (Navarrete, 2008).

En contraste con el proceso de franca declinación que padece Comayagua en el siglo XIX, Tegucigalpa se fortalece. El hecho de no ser capital la libró de los teatros de conflictos bélicos que asolan la post independencia centroamericana. $\mathrm{La}$ actividad minera, así como en su origen, fue el motor que desencadenará el traslado de la capital del Estado. Desde el año 1878 el presidente Soto y el empresario neoyorquino Julius Valentine organizan y capitalizan la New York \& Rosario Mining Company, constituyéndola en 1879. El año siguiente la compañía recibió una concesión de veinte años renovables para que explote una mina en los alrededores de la ciudad: las minas del Rosario en San Juancito. El presidente Soto calculó el traslado de capital a Tegucigalpa desde su llegada a la presidencia, como se lee en el decreto de traslado de la capital que:

Considerando que la ciudad de Tegucigalpa reúne las condiciones $i$ elementos necesarios de población $i$ riqueza, para la residencia del gobierno $i$ de la corte suprema de justicia $\mathrm{i}$ reunión del congreso, que en ella se encuentran el almacén principal de guerra la casa de la moneda i la imprenta nacional. Lo mismo que las oficinas centrales de renta, telegráficas $i$ de correos por tanto, decreta,

Artículo único: se declara la ciudad de Tegucigalpa, por ahora, capital de la república (Asamblea Nacional Constituyente, 30 de octubre de 1880) $)^{4}$.

Daniela Navarrete Cálix 
Tegucigalpa se consagra con la primacía urbana frente el resto del territorio hondureño. Este decreto argumentó tres puntos claves: primero, el decreto es emitido por la Asamblea Nacional Constituyente, lo que imprime mayor legitimidad y fuerza a una disposición emanada de los representantes del pueblo para la delicada misión de crear una nueva constitución. Segundo, el título del decreto declara a Tegucigalpa capital de la república por abora, lo que pudo distraer el recelo de parte de los desconfiados comayagüenses. Y tercero, el presidente Soto se encargó desde que asumió la primera magistratura del país en 1876 en potenciar la infraestructura existente en Tegucigalpa y poner en marcha los modernos servicios necesarios para la nueva función: sedes para los tres poderes del estado, almacén de guerra, casa de la moneda, imprenta, los servicios recién organizados de telecomunicaciones, correos además de la existencia desde 1847 de la Universidad Nacional. Soto supo preparar el terreno para el traslado, secundado por la Asamblea y calmando los opositores con la promesa de la transitoriedad. El traslado de la capitalidad constituye un momento mayor en el ascenso urbano de Tegucigalpa y en la vida de sus habitantes. En tanto, también tuvo un fuerte impacto negativo para los habitantes de la antigua capital, quienes se opusieron al traslado. ¿Cómo se desmonta una capital?, ¿`ómo se monta una nueva? Este fenómeno ha sido estudiado para el caso brasileño por Laurent Vidal (2009), cuando se trasladó la capital de Río de Janeiro a Brasilia en 1960.
Para Tegucigalpa, la organización de la puesta en escena de la nueva capital política y administrativa tuvo por epicentro los espacios públicos: creando nuevos paseos y avenidas, así como la reconversión de las plazas coloniales en parques republicanos. Se sumaron a los actos veladas culturales, en la usanza de tertulias patrióticas, colocación de primeras piedras para edificios públicos, etc. Todos estos eventos procuraron la modernización de la ciudad. Para los comayagüenses, no quedó más que el recurso desesperado e ingenuo de sabotear la mudanza de las oficinas públicas, escondiendo las llaves de las puertas o protestando para impedir el traslado de mobiliario. Con ocasión de la creación del Archivo Nacional a inicios de 1880 - meses antes del decreto de traslado de capital-, el primer director de la institución, Antonio R. Vallejo, procedió a mudar los archivos estatales de Comayagua:

Después pasamos a registrar el [archivo] de la ciudad de Comayagua, y para no herir el sentimiento local, tan pronunciado en sus habitantes, pedíamos secretamente la llave al señor Gobernador político. A la diez de la noche $[. .$.$] donde se encontraban la multitud$ de papeles $[. .$.$] los acomodábamos en sacos$ y matates [...] y a las cuatro de la mañana los hacíamos cargar en bestias... esta operación se ejecutó en varias ocasiones, sin que nadie supiera que transportábamos el archivo de la capital a la ciudad de Tegucigalpa (Vallejo, 1880 en Reina, 1980, p. 330 $)^{5}$. 
El crecimiento económico derivado de la exportación minera, no conllevó en Tegucigalpa una explosión demográfica como en otras urbes industrializadas de América Latina (ver tabla 1). En contraste, el crecimiento vertiginoso de las ciudades bananeras del norte, lideradas por San Pedro Sula, situó a esta ciudad como competidora por la primacía urbana del país.

\section{3. ¿Dicotomía por la primacía o la modernidad? Tegucigalpa y San Pedro Sula (1920-1990)}

El principal rubro de exportación del Estado liberal hondureño cambió a inicios del siglo XX. En la costa norte del país se otorgaron grandes concesiones de territorio a compañías norteamericanas para que cultivaran banano. En la segunda década del siglo las exportaciones de banano desplazan por completo las de la minería. Toda la región atlántica es revitalizada con infraestructura de comunicaciones (puertos, ferrocarril, carreteras, ciudades nuevas) lo que socava la hegemonía simbólica y económica de Tegucigalpa, epicentro del eje centro-sur desde tiempos hispánicos. Aunque lejos de los puertos bananeros, Te- gucigalpa consigue mantener su primacía urbana frente a las dos principales sedes bananeras: La Ceiba y, sobretodo, San Pedro Sula. Lo anterior gracias a los poderes políticos que concentra (sede del ejecutivo, judicial y legislativo) y a la capacidad de canalizar los aportes económicos que las empresas bananeras vertían para lograr el respaldo gubernamental. También por la fuerte voluntad política de los gobernantes como Soto, Bográn, Bonilla, Bertrand y Carías al fortalecer la infraestructura pública, comunicaciones, educativa, cultural y de servicios de la capital.

Aún con todo el impulso que recibe Tegucigalpa en el siglo XX, lo cierto es que sus capacidades simbólicas son opacadas por las de San Pedro Sula. En menos de medio siglo, esta población pasó de un villorrio atrasado a ser la principal ciudad costera, como lo describe Vallejo en su anuario de 1889:

El aspecto físico de esta población es importante y cuenta con un comercio floreciente, una sociedad culta y activa, y con elegantes y cómodos edificios, construidos al estilo moderno. La choza de paja del indígena, ha cedido allí el puesto a los edificios formales

\section{territarias 37}

Tabla 1. Población en los principales centros urbanos hondureños 1881-1974

\begin{tabular}{|l|c|c|c|c|c|c|c|}
\hline Ciudad/Hab & $\mathbf{1 8 8 1}$ & $\mathbf{1 9 0 0}$ & $\mathbf{1 9 3 0}$ & $\mathbf{1 9 3 5}$ & $\mathbf{1 9 5 0}$ & $\mathbf{1 9 6 1}$ & $\mathbf{1 9 7 4}$ \\
\hline Tegucigalpa & 12000 & 24000 & 40695 & 47760 & 90613 & 154429 & 304891 \\
\hline San Pedro Sula & & & 28327 & 33720 & 45392 & 94474 & 208083 \\
\hline Total & & & 69022 & 81480 & 136005 & 248903 & 512974 \\
\hline
\end{tabular}

Fuente: Instituto Nacional de Estadísticas de Honduras, (s.f.). 
cubiertos de pizarra y otros de zinc. Posee un extenso y hermoso cabildo recientemente construido (Vallejo, 1997, p. 86).

Desde el período hispánico, el destino de esta villa (1524) estaba ligado a la actividad portuaria de Puerto Caballos. El desuso de éste último en el siglo XVIII conllevó a su casi desaparición. Pero cuando el gobierno republicano restituyó el Puerto Cortés y se creó el departamento homónimo en 1893, se fortalecieron las capacidades políticas de San Pedro al nombrarla cabecera. En lo económico, además del puerto se comenzó la construcción del ferrocarril interoceánico en la década de 1860 y se construyen carreteras a finales del siglo XIX. No solo el banano desplaza la minería, el departamento de Cortés, industrializado también, provee el $30 \%$ del PIB para 1950. San Pedro Sula articula la región atlántica del país, integrando campos de cultivo para exportación, zonas industriales con una serie de ciudades nuevas, creadas inicialmente por el esfuerzo urbanizador del gobierno y su proyecto de ferrocarril. Se crearon nuevos departamentos: Colón (1883), Cortés (1893) y Atlántida (1902). Las ciudades y comunidades de estos departamentos prosperaron rápidamente gracias a la actividad generada por tres compañías bananeras: la United Fruit Co., la Cuyamel Fruit Co. y la Standard Fruit Co. Estas empresas marcaron visiblemente el paisaje urbano regional. Las sedes locales de estas empresas se construyeron al estilo de enclave en que operaba su economía. Los pueblos de la compañía o company towns se levantaron en áreas restringidas por cercos y muros en las periferias de ciudades existentes. Su funcionamiento era al estilo gated community, apartheid urbano que dura hasta nuestros días en localidades como La Lima. Otras fueron La Ceiba, El Progreso y Tela, todas estas ciudades se volvieron faro de la modernidad, irradiando nuevas estéticas constructivas, símbolo de los valores liberales: el progreso técnico y la prosperidad económica.

A finales del siglo XX, el fenómeno de desplazamiento de la primacía urbana de Tegucigalpa se volvió a acentuar. En el marco de la aplicación de doctrinas neoliberales, los financiamientos internacionales y nacionales eludieron a la capital, los primeros para desestimular las migraciones del campo y los segundos para privilegiar la región del valle de Sula y las maquilas. La inversión del Estado se concentró en reforzar la infraestructura productiva y de comunicaciones de la región del valle de Sula. Tal inversión, en vías de comunicación terrestre, marítima y aérea, legislación a favor de zonas de industriales de procesamiento (ZIP) o maquilas, propició la aparición del primer conurbano en Honduras, articulado por la ciudad de San Pedro Sula y otras como El Progreso, La Lima, Puerto Cortés, Choloma y Villanueva, entre otras. Esta vez, a diferencia del tiempo del boom bananero, Tegucigalpa, ya sin minería ni puerto en Amapala ${ }^{6}$, no pudo competir y fue abandonada por el Estado, que dejó la Municipalidad de la capital sin autonomía real y como botín político.
${ }^{6}$ Honduras mantiene un puerto en el Pacífico, El Henecán, cuya apertura propició el declive de Amapala.

tersitarios 37 
La cara del abandono estatal en la capital es la pobreza, que alcanzó en esos años amplitudes industriales. Para el historiador Marcos Carías (2010), en la sociedad post moderna la marginalidad se vuelve masiva, anónima y excluyente.

Otro efecto en la ciudad de las medidas neoliberales, fue la manera de (de)construir la ciudad, desde entonces en manos de los mecanismos de mercado. Para esos años el ritmo de crecimiento de Tegucigalpa ha llegado a duplicar su población cada veinte años. La población en 1988 es de 750000 habitantes, de los cuales el $60 \%$ viven en asentamientos ilegales, con pocos o ningún servicio público y, más dramático aún, en zonas de alto riesgo de inundaciones o derrumbes. Para 1996, en la capital solo el $60 \%$ de la población tiene servicio público de agua potable instalado, $22 \%$ se abastece con privados o conexión ilegal y $18 \%$ no tiene acceso. Con estos retrocesos, en los 90 las condiciones están dadas para que los problemas de la suburbanización, como les llama Davis (2005), exploten en las periferias.

El impacto de la pobreza urbana en Honduras no es un fenómeno exclusivo de Tegucigalpa, pero su topografía accidentada la visibiliza más en contraste con el valle del Ulúa donde se encuentra San Pedro Sula. Precisamente esta ventaja geográfica le facilita las vías de comunicación modernas, tanto terrestres como aéreas. El mejor aeropuerto comercial del país es el Villeda Morales mismo que tiene rango internacional, el único país centroamericano en esos años en tener esta dualidad aeroportuaria. Esta facilidad de comunicaciones con EE.UU. permitió a las élites sampedranas tener una conexión constante con ciudades como Miami, Houston o Nueva Orleans con sus aviones privados. Las autopistas, esquemas de planificación urbana y facilidades, suman en la competitividad a favor de esta ciudad, siendo el aeropuerto la infraestructura clave entre todas. Especialmente, cuando el aeropuerto Toncontín, en contraposición, es la infraestructura que más controversia genera en Tegucigalpa. Construido a inicios de siglo XX, desde los años 50 con el impacto de las tecnologías aéreas (de motor de hélice a propulsión con turbinas), se identificó la necesidad de su ampliación o su traslado. En los años 80 , en el contexto de la Guerra Fría y la colaboración militar hondureña con los EE.UU. (el apoyo a la Contra nicaragüense y a los ejércitos salvadoreños, guatemaltecos y hondureños) se construyó en Comayagua la base militar Soto Cano, más conocida como Palmerola. Por su cercanía, este aeropuerto funcionó como subsidiario de Tegucigalpa. Por ejemplo, cuando el Papa Juan Pablo II visitó Tegucigalpa en 1981, el avión que lo trasladaba tuvo que aterrizar en Palmerola pues la pista de $2 \mathrm{~km}$ de largo de Toncontín no garantizaba un aterrizaje seguro. No solo la pista representaba un peligro, lo es también su emplazamiento rodeado de montañas. Por lo menos una media docena de incidentes se habían presentado desde los años 60, cuando en 1989, la aerolínea hondureña tersitarias 37 
SAHSA tuvo el peor accidente de la historia aeronáutica hondureña. Un avión Boeing 727 , se estrelló en las montañas adyacentes, matando a 131 de los 149 pasajeros. Más recientemente, el descarrilamiento de un avión de la aerolínea salvadoreña TACA en el 2008 provocó que el presidente de Honduras decretara el cierre de Toncontín. La ciudadanía estaba dividida ante la decisión, hasta que el alcalde de la capital tomó el liderazgo de oponerse a la medida por las pérdidas económicas que traería, así como el colapso simbólico de Tegucigalpa. A raíz de estos acontecimientos, los gobiernos nacionales sucesivos han impulsado la construcción de un aeropuerto internacional en Comayagua, usando la pista de la base militar Soto Cano/Palmerola. La medida ha generado oposición en amplios sectores de la capital, que ven inminente el cierre de Toncontín una vez iniciada las operaciones en Comayagua. Los gremios más afectados son los empleados directos e indirectos del aeropuerto: taxistas, cargadores, restaurantes, servicios, comercios, casas de cambio, etc. Pero también los políticos se dan cuenta de la amenaza clara a la primacía de Tegucigalpa, especialmente los de oposición, quienes se presentan en debates públicos para rechazar la medida. En el actual contexto de gobierno, con mayoría oficialista en la Asamblea General, no deja muchas posibilidades de retorno. En cambio, son las razones presupuestarias - el costo estimado de la obra se eleva a US \$300 millones - las que postergan la medida, por ahora.

\section{Conclusiones}

En los tres momentos estudiados, encontramos diferentes motores o causales que motivaron estos conflictos interurbanos. En el primer momento (1788-1812), la supresión de la jurisdicción mayor (la Alcaldía Mayor) que sustenta económica y políticamente a la Villa de Tegucigalpa, fue providencial a los intereses de las élites de Comayagua. La implementación del régimen de Intendencias permitió a la capital de provincia limitar las capacidades de la nueva villa, que fue identificada por la corona misma como potencial capital. La lucha por la restitución de la Alcaldía fue el antecedente directo ante la opción de independencia de España. Las represalias de Comayagua, que incluyeron persecución política de vecinos tegucigalpenses y obstaculización del título de ciudad, no impidieron que el conflicto fortaleciera a los vecinos a través de alianzas extraprovinciales.

Esto se materializó en el momento de la Independencia, cuando el cabildo de Guatemala reconoce a su aliada otorgándole el anhelado título de ciudad. Tegucigalpa reúne todos los méritos (políticos, económicos y urbanos) que el Antiguo Régimen señalaba indispensables para ser la capital primada de una provincia y subdivisiones. En el segundo momento (1821-1880), el conflicto evoluciona de la subordinación económica y política a la lejana metrópoli, a la pugna por remplazar a la Corona en las lealtades de los nuevos ciudadanos. 
Comayagua es nuevamente derrotada, tras largas décadas de desgaste por guerras civiles y fenómenos naturales, perdiendo sus capacidades como emisora de modernidad urbana. El traslado de la capital a Tegucigalpa cierra el capítulo, pero en pocos años emerge un nuevo contrincante. El proyecto liberal emprendido desde el s. XIX y que fortaleció la minería de exportación en los alrededores de Tegucigalpa, no fructifica lo suficiente comparado con las proporciones alcanzadas por la expansión económica de las bananeras y la emergente red urbana liderada por San Pedro Sula. En el tercer momento (1920-1990) la dicotomía urbana hondureña se reabre con San Pedro Sula. En este conflicto, la modernidad liberal impone el parámetro económico sobre el político. Importa más tener una red de comunicaciones eficiente con los mercados mundiales, que albergar las sedes del gobierno nacional. A la fecha las autoridades sampedranas no han emprendido acción oficial para rivalizar por la sede política del país. En los últimos años se ha anunciado la apertura de un nuevo y mejor aeropuerto en Comayagua y el cierre de Toncontín, lo que desencadenó la reacción de los tegucigalpenses que temen la pérdida de la competitividad de la capital. Estas nuevas dinámicas urbanas, donde no se disputan jerarquías como en tiempos hispánicos, revelan un cambio de modelo urbano más equilibrado donde dos o más ciudades grandes contienen las fuerzas urbanas del país. Los tiempos del anhelo de ciudad quedan bien atrás, así como el de las rivalidades por estatutos de prestigio simbólico. Los nuevos atributos de la modernidad urbana lo confieren ya no los títulos reales sino las capacidades materiales de comunicación ligados por supuesto a la eficiencia y competitividad de la economía de mercado.

\section{Referencias}

Anales del Archivo Nacional de Honduras. (julio, 1868). Decreto N. ${ }^{\circ} 11$ en que se declara la ciudad de Tegucigalpa por abora capital de la República.

Barahona, M. (1993). La alcaldía mayor de Tegucigalpa bajo el régimen de Intendencias (1788-1812), Tegucigalpa: IHAH.

Brading, D. (1991). Orbe indiano. México D.F.: Fondo de Cultura Económica.

Cabildo de Tegucigalpa. (1806). Acta capitular del 24 de septiembre de 1806. Archivo Municipal de Tegucigalpa (Libro de actas capitulares y de elecciones 1801-1837), Tegucigalpa, Honduras.

Calvo, T. (1995). L'Amérique ibérique de 1570 à 1910. París: Nathan.

Carías, M. (2010). De la patria del criollo a la patria compartida. Choluteca: Subirana.

Davis, M. (2005). Planéte bidonvilles. París: Ab irato.

De Solano, F. (1987). Historia urbana de Iberoamérica. Madrid: Consejo Superior de los Colegios de Arquitectos de España.

De Tornos, A. (1815). Informe del Gobernador Intendente de Comayagua Antonio de Tornos. Archivo General de

\section{territarias 37}


Indias (Audiencia de Guatemala, Legajo \#973), Sevilla, España.

Guerra, F. X.(1993). Modernidad e independencias. Ensayos sobre las revoluciones hispánicas. México D.F.: Fondo de Cultura Económica \& MAPFrE.

Gruzinsky, S. (1999). La pensée métisse. París: Fayard.

Kinloch, F. (2005). Conflictos limítrofes y discurso nacionalista. La frontera $\mathrm{Ni}$ caragua-Costa Rica (1824-1858). En P. Bovin (Dir.), Fronteras y sociedades entre el sur de México y América Central (pp. 97-107). México D.F.: CEMCACIESAS.

Lester, M. (1971). Un viaje por Honduras. San José: EDUCA.

MacLeod, M. (1976). Socio economic history of Spanish Central America. Los Angeles: Berkeley.

Martínez, M. F. (1982). Apuntamientos para una historia colonial de Tegucigalpa y su Alcaldia Mayor. Tegucigalpa: Editorial Universitaria.

Martínez, S. (1807). Petición del apoderado del ayuntamiento de la Villa de Tegucigalpa para solicitar el título de ciudad. (Legajo \#14 1100-1107). Archivo Municipal de Guatemala, Guatemala.

Musset, A. (2011). Ciudades nómadas del Nuevo Mundo. México D.F.: Fondo de Cultura Económica.
Navarrete, D. (2008). Evolución urbana de Comayagua 1537-1975. Tegucigalpa: IHAH.

Reif, S. J. (1980). Comayagua a city in Central America (Tesis de doctorado sin publicar), Universidad Estatal de Ohio, Columbus, EE.UU.

Reina, J. (1980). Historia de Tegucigalpa. Tegucigalpa: AMDC.

Squier, E. G. (1971). Notes on Central America. Particulary the status of Honduras and San Salvador 1855. Nueva York: Ams Press Inc.

Taracena, L. P. (1998). Ilusión minera y poder politico, la Alcaldia Mayor de Tegucigalpa siglo XVIII. Tegucigalpa: Guaymuras.

Tinoco, J. G. (1820). Carta del Gobernador Intendente José Gregorio Tinoco de Contreras al Rey informando sobre su provincia. (Audiencia de Guatemala, Legajo \#531), Archivo General de Indias, Sevilla, España.

Vallejo, A. (1997). Primer anuario estadistico 1889. Tegucigalpa: Editorial Universitaria.

Vidal, L. (2009). Les larmes de Rio le dernier jour d'une capitale. París: Flammarion/ Auber.

Zepeda, I. (2006). La familia Midence, una historia vigente. Tegucigalpa: Lithopress. territarias 37

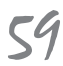

